\title{
The effects of palm kernel cake based diet on spermatogenesis in Malin X Santa-Ines rams
}

\begin{abstract}
Testes from nine male Malin $\times$ Santa-Ines rams with an average body weight of $43.1 \pm 3.53$ $\mathrm{kg}$, were used to study the effects of palm kernel cake (PKC) based diet on spermatogenic cells and to assess copper $(\mathrm{Cu})$ levels in liver, testis and plasma in sheep. Animals were divided into three groups and randomly assigned three dietary treatments using restricted randomization of body weight in completely randomized design. The dietary treatments were $60 \%$ palm kernel cake plus $40 \%$ oil palm frond (PKC), $60 \%$ palm kernel cake plus $40 \%$ oil palm frond supplemented with $23 \mathrm{mg} / \mathrm{kg}$ dry matter of molybdenum as ammonium molybdate [(NH4)6Mo7O24.4H2O] and $600 \mathrm{mg} / \mathrm{kg}$ dry matter of sulphate as sodium sulphate [Na2SO4] (PKC-MS) and 60\% concentrate of corn-soybean mix $+40 \%$ oil palm frond (Control), the concentrate was mixed in a ratio of $79 \%$ corn, $20 \%$ soybean meal and $1 \%$ standard mineral mix. The results obtained showed that the number of spermatogonia, spermatocytes, spermatids and Leydig cells were not significantly different among the three treatment groups. However, spermatozoa, Sertoli cells and degenerated cells showed significant changes, which, may be probably due to the $\mathrm{Cu}$ content in PKC. Liver and testis $\mathrm{Cu}$ levels in the rams under $\mathrm{PKC}$ diet was found to be significantly higher $(\mathrm{P}<0.05)$ than rams in Control and PKC-MS diets. Plasma $\mathrm{Cu}$ levels showed a significant increase $(\mathrm{P}<$ 0.05) at the end of the experiment as compared to at the beginning of the experiment for PKC and Control. In conclusion, spermatogenesis is normal in rams fed the diet without PKC and PKC supplemented with Mo and S. However spermatogenesis was altered in the PKC based diet probably due to the toxic effects of $\mathrm{Cu}$ and the significant changes in organs and plasma. Thus, Mo and $\mathrm{S}$ play a major role in reducing the accumulation of $\mathrm{Cu}$ in organs.
\end{abstract}

Keyword: Ammonium molybdate, Copper toxicity, Palm kernel cake, Rams, Spermatogenesis 